\title{
Photoevaporation of Circumstellar Disks Revisited: The Dust-Free Case
}

\author{
Kei E. I. Tanaka ${ }^{1,2,3}$, Taishi Nakamoto ${ }^{3}$, and Kazuyuki Omukai ${ }^{1,2}$ \\ 1: Astronomical Institute, Tohoku University, Sendai 980-8578, Japan \\ 2: Department of Physics, Kyoto University, Kyoto 606-8502, Japan \\ 3: Department of Earth and Planetary Sciences, Tokyo Institute of Technology, Tokyo 152-8551, Japan \\ ktanaka@astr.tohoku.ac.jp
}

\begin{abstract}
Photoevaporation by stellar ionizing radiation is believed to play an important role in the dispersal of disks around young stars. The mass loss model for dust-free disks developed by Hollenbach et al. is currently regarded as a conventional one and has been used in a wide variety of studies. However, the rate in this model was derived by the crude so-called $1+1 \mathrm{D}$ approximation of ionizing radiation transfer, which assumes that diffuse radiation propagates in a direction vertical to the disk. In this study, we revisit the photoevaporation of dust-free disks by solving the 2D axisymmetric radiative transfer for steady-state disks. Unlike that solved by the conventional model, we determine that direct stellar radiation is more important than the diffuse field at the disk surface. The radial density distribution at the ionization boundary is represented by the single power-law with an index $-3 / 2$ in contrast to the conventional double power-law. For this distribution, the photoevaporation rate from the entire disk can be written as a function of the ionizing photon emissivity $\Phi_{\mathrm{EUV}}$ from the central star and the disk outer radius $r_{\mathrm{d}}$ as follows: $\dot{M}_{\mathrm{PE}}=5.4 \times 10^{-5}\left(\Phi_{\mathrm{EUV}} / 10^{49} \mathrm{sec}^{-1}\right)^{1 / 2}\left(r_{\mathrm{d}} / 1000 \mathrm{AU}\right)^{1 / 2} M_{\odot} \mathrm{yr}^{-1}$. This new rate depends on the outer disk radius rather than on the gravitational radius as in the conventional model, caused by the enhanced contribution to the mass loss from the outer disk annuli. In addition, we discuss its applications to present-day as well as primordial star formation.
\end{abstract}

Subject headings: Stars: formation - Stars: Population III - Stars: massive - Radiative transfer - Accretion, accretion disks - Protoplanetary disks - (ISM:) HII regions

\section{Introduction}

Stars are formed by the gravitational collapse of pre-stellar cores with non-zero angular momentum. As a natural results, disks are formed around newborn stars, and most of the materials are accreted through thses. The final stellar mass at the time of its formation is set when the disk dissipates. Moreover, disk dissipation determines the formation environments of planets, which are formed inside disks at the final stage of low-mass star formation. Photoevaporation is currently considered to be a promising dissipation mechanism by which the disk gas escapes from the gravitational binding of a star as a result of heating by ultraviolet (UV) radiation from the star or external sources. While the irradiation by nearby stars can be important in the case of low-mass star formation in a dense cluster, (e.g., Johnstone et al. 1998; Adams et al. 2004; Fatuzzo \& Adams 2008; Holden et al. 2011; Thompson 2013), the photoevaporation by radiation from the central star should dominate in isolated or massive star formation. In this study, we focus on the latter process.

The photoevaporation of protoplanetary disks around low-mass stars has been studied since 1990s. Early studies concentrated on photoevaporation by extreme ultraviolet (EUV), i.e., ionizing radiation (Shu et al. 1993; Clarke et al. 2001) from the central star. However, photo- 
evaporation by far-ultraviolet (FUV) radiation and/or X-rays is now considered as a more dominant mechanism for disks around low-mass stars (Gorti \& Hollenbach 2009; Owen et al. 2012) on the basis that its timescale to operate, $\sim 3 \mathrm{Myr}$, is consistent with the observational disk-dissipation timescale. However, this theory remains in dispute.

EUV photoevaporation has attracted significant attention recently in the context of primordial star formation in the early universe. Although primordial stars were first speculated to be $\sim 1000 M_{\odot}$ because of their natal pre-stellar core masses (Bromm et al. 1999; Omukai \& Palla 2003), recent studies that consider stellar feedback onto accretion flows via the disk demonstrated that the EUV effect on the infalling gas becomes significant for masses greater than $10 M_{\odot}$, and that photoevaporation terminates mass accretion onto the newborn star at a mass of $20-100 M_{\odot}$ (McKee \& Tan 2008; Hosokawa et al. 2011, 2012; Stacy et al. 2012). A similar mechanism can be applied to present-day massive star (> $\left.20 M_{\odot}\right)$ formation, although this topic has not been studied in depth thus far.

In studies of disk evolution by EUV photoevaporation, the formula derived by Hollenbach et al. (1994) (hereafter, HJLS94) has often been used. By calculating the approximate radiative transfer (RT) for assumed steady-state density distributions around circumstellar disks, HJLS94 derived the mass-loss rate from dust-free disks as

$$
\begin{aligned}
& \dot{M}_{\mathrm{PE}, \mathrm{H} 94}=1.3 \times 10^{-5} \text { isequalto }\left(\frac{\Phi_{\mathrm{EUV}}}{10^{49} \mathrm{~s}^{-1}}\right)^{1 / 2} \\
& \times\left(\frac{M_{*}}{10 M_{\odot}}\right)^{1 / 2} M_{\odot} \mathrm{yr}^{-1},
\end{aligned}
$$

where $\Phi_{\mathrm{EUV}}$ is the EUV photon emission rate, and $M_{*}$ is the mass of the central star. Although HJLS94 emphasized the importance of diffuse radiation, i.e., the re-emitted radiation from the ionized atmosphere above the disk, in ionizing the disk surface, such radiation was not adequately treated in their calculation. To save computational expence, they adopted the $1+1 \mathrm{D}$ approximation for RT, where diffuse radiation is assumed to propagate in a direction vertical to the disk. In reality, of course, diffuse radiation also has radial components, because the density and ionization degree have radial gradients in the atmosphere. In this study, to accurately treat diffuse radiation, we calculate the axisymmetric 2D RT and re-examine the results of HJLS94.

In Section 2, we describe the basics of photoevaporation and our model for calculation. In Section 3 , we present our results of the 2D RT calculation, which is significantly differs from the previous $1+1 \mathrm{D}$ result. In Section 4, we analytically interpret the numerical results and derive a new formula for the photoevaporation rate for dustfree disks. Section 5 is reserved for a discussion on our model's implication on star formation and its validity and limitations. Finally, we summarize our study in Section 6.

\section{Model}

Photoevaporation is a mass-loss process from a circumstellar disk due to radiative heating either by its central star or by external objects (Bally \& Scoville 1982). We evaluate the massloss rate that results from EUV heating by the central star. Below, we first describe the basics of photoevaporation; Figure 1 presents a schematic view of our model. We use the axisymmetric cylindrical coordinates $R$ for the distance from the symmetric axis and $Z$ for the height from the equatorial plane.

\subsection{Photoevaporation}

We consider photoevaporation from the disk around a star with mass $M_{*}$. Because the density above the disk is lower than that in the equatorial plane, EUV radiation irradiates the upper surfaces of the disk, and the irradiated thin surface of the disk is ionized. The ionized gas is heated to a temperature of $\sim 10^{4} \mathrm{~K}$, which increases the sound speed to $c_{\mathrm{s}, \mathrm{HII}} \approx 10 \mathrm{~km} \mathrm{~s}^{-1}$, and forms the $\mathrm{H}$ II region above the disk. The characteristic scale length, the so-called gravitational radius, is defined as the radius in which the Keplerian velocity ( $\simeq$ escape velocity) is equal to $c_{\mathrm{s}, \mathrm{HII}}$,

$$
r_{\mathrm{g}}=\frac{G M_{*}}{c_{\mathrm{s}, \mathrm{HII}}^{2}} \simeq 70\left(\frac{M_{*}}{10 M_{\odot}}\right) \mathrm{AU} .
$$

Inside the gravitational radius, $r \lesssim r_{\mathrm{g}}$, the ionized gas is gravitationally bound and an ionized atmosphere is created above the un-ionized disk. However, outside the gravitational radius, $r \gtrsim r_{\mathrm{g}}$, 


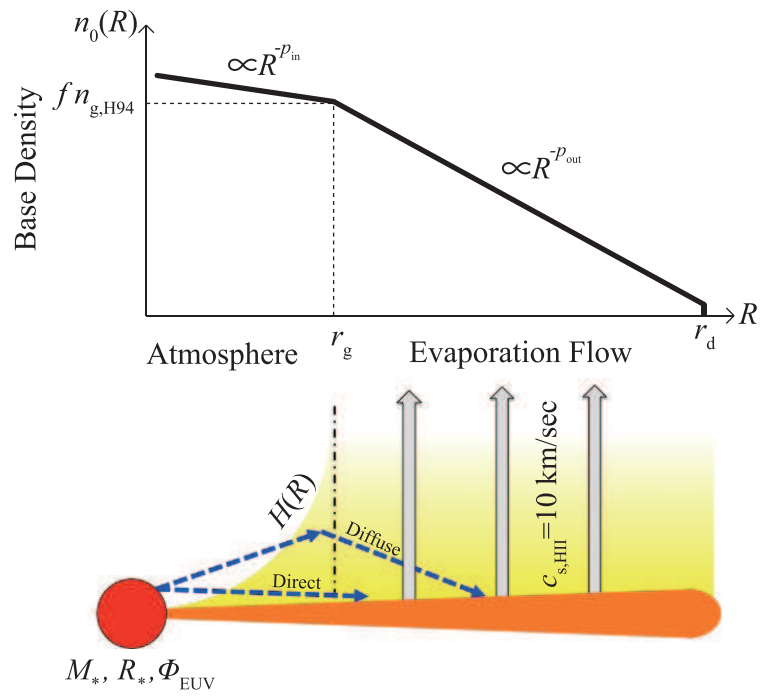

Fig. 1. - Schematic view of our model. Bottom: Direct stellar radiation and diffuse radiation from recombination irradiate and ionize the disk surface, forming the atmosphere $\left(R<r_{\mathrm{g}}\right)$ and the evaporation flow $\left(R>r_{\mathrm{g}}\right)$. We assume a zerothickness neutral disk, although the disk in this schematic view has finite thickness for illustration purposes. Top: Piecewise power-law distribution for the number density at the bottom of the $\mathrm{H}$ II region, or the base density (equations. 8] 10]).

the thermal energy of the ionized gas is greater than its gravitational energy; thus, the ionized gas is unbound. Therefore, the ionized gas flows away from the disk surface, and the gas is photoevaporated. Because the photoevaporation flow is driven by thermal pressure, the flow velocity is approximately given by the sound speed $c_{\mathrm{S}, \mathrm{HII}}$. Although the definition of the gravitational radius slightly changes if hydrodynamic effects such as gas pressure, radiation pressure, and angular momentum are included (Liffman 2003; Font et al. 2004; McKee \& Tan 2008), we here use eq.(2) as the gravitational radius for easier comparison with HJLS94. Our results in Section 4 later show that the photoevaporation rate does not depend on the gravitational radius. This justifies our approximation for $r_{\mathrm{g}}$.

Because the photoevaporation flow velocity is $\simeq c_{\mathrm{s}, \mathrm{HII}}$, the mass-loss flux per unit area at radius
$R$ is

$$
\dot{\Sigma}_{\mathrm{PE}}(R)=m_{\mathrm{H}} c_{\mathrm{s}, \mathrm{HII}} n_{0}(R),
$$

where $m_{\mathrm{H}}$ is the proton mass, and $n_{0}(R)$ is the number density at the bottom of the ionization layers, which is the boundary of the ionized H II region and the unionized disk. We hereafter refer to $n_{0}(R)$ the base density. The evaporation flow launches from the disk outside $r_{\mathrm{g}}$ to form an annulus with $r_{\mathrm{g}}<R<r_{\mathrm{d}}$, where $r_{\mathrm{d}}$ is the outer radius of the disk. By summing the contribution from both the upper and lower surfaces, the total photoevaporation rate from the disk is

$$
\begin{aligned}
\dot{M}_{\mathrm{PE}} & =2 \int_{r_{\mathrm{g}}}^{r_{\mathrm{d}}} 2 \pi R \dot{\Sigma}_{\mathrm{PE}}(R) d R \\
& =4 \pi m_{\mathrm{H}} c_{\mathrm{s}, \mathrm{HII}} \int_{r_{\mathrm{g}}}^{r_{\mathrm{d}}} n_{0}(R) R d R .
\end{aligned}
$$

For a given base density distribution $n_{0}(R)$, we can calculate the photoevaporation rate. HJLS94 estimated the base density distribution from the RT calculation with the $1+1 \mathrm{D}$ approximation and derived the photoevaporation rate in equation (11). In this study, we repeat a similar analysis by using the 2D RT calculation.

\subsection{Ionized gas structure}

Following HJLS94, we assume that (1) the steady-state density distribution and (2) the thin neutral gas disk. From these assumptions, the ionization front is located at $Z=0$. We confirmed that our results with this thin-disk approximation are consistent with the hydrodynamic simulation for a finite-thickness disk (Section 5.2). Although the exact temperature of the ionized gas slightly depends on detailed heating and cooling processes, we retained the typical value of $T_{\mathrm{HII}}=10^{4} \mathrm{~K}$.

\subsubsection{Vertical structure}

Inside the gravitational radius $\left(R<r_{\mathrm{g}}\right)$, the bound ionized gas forms an atmosphere above the disk. The gas is vertically hydrostatic in this region,

$$
n(R, Z)=n_{0}(R) \exp \left(-\frac{Z^{2}}{2 H^{2}}\right)
$$

where the scale height is expressed as

$$
H(R)=\frac{c_{\mathrm{s}, \mathrm{HII}}}{\Omega_{\mathrm{K}}}=r_{\mathrm{g}}\left(\frac{R}{r_{\mathrm{g}}}\right)^{3 / 2} .
$$


On the contrary, the ionized gas will evaporate away in an outer flow region $\left(R>r_{\mathrm{g}}\right)$. In this region, we assume that the density is vertically constant,

$$
n(R, Z)=n_{0}(R) .
$$

Although this assumption fails in the upper region of $Z>R$ as shown in the hydrodynamic simulation by Font et al. (2004), we apply the concept for simplicity and for a comparison with HJLS94. In consideration of our result that the density distribution in the lower layers of $Z \ll R$ is more important, this assumption dose not significantly affect our conclusion.

\subsubsection{Radial structure}

The vertical structure of the ionized region, which includes the atmosphere and evaporation flow, is given by equations (6) and (7). To specify the radial density distribution, we need the base density distribution, $n_{0}(R)=n(R, Z=0)$. Here we suppose that the disk is neutral and geometrically thin and that the ionized gas pervades above the neutral disk. Thus, for a plausible density profile, the ionization front must coincide with the disk surface at $Z=0$. With densities higher than the plausible value, a neutral zone appears above the disk. This situation is inconsistent, because the vertical structure at $Z>0$ is only valid for hot ionized gas (equations 6 and 7 ). On the contrary, if the density is very low, ionizing photons can reach the disk and ionize its upper layer, which also conflicts with our assumption of the neutral gas disk. In the following equation, by calculating the ionization structure for various base-density profiles, we search for the plausible density profile, which places the ionization front at $Z=0$.

In searching for the plausible base-density profile, we assume the piecewise power-law distribution with different exponent indices inside or outside the gravitational radius as in HJLS94:

$$
n_{0}(R)=f n_{\mathrm{g}, \mathrm{H} 94}\left(\frac{R}{r_{\mathrm{g}}}\right)^{-p},
$$

where

$$
\begin{aligned}
n_{\mathrm{g}, \mathrm{H} 94}=1.8 & \times 10^{7}\left(\frac{\Phi_{\mathrm{EUV}}}{10^{49} \mathrm{~s}^{-1}}\right)^{1 / 2} \\
& \times\left(\frac{r_{\mathrm{g}}}{10^{15} \mathrm{~cm}}\right)^{-3 / 2} \mathrm{~cm}^{-3},
\end{aligned}
$$

$$
p= \begin{cases}p_{\text {in }} & \text { for } R<r_{\mathrm{g}}, \\ p_{\text {out }} & \text { for } R>r_{\mathrm{g}},\end{cases}
$$

where $f, p_{\text {in }}$, and $p_{\text {out }}$ are dimensionless parameters. With the parameters set to $f=0.9, p_{\text {in }}=$ 1.5 , and $p_{\text {out }}=2.5$, our base density reproduces that of HJLS04 model. The normalization density $n_{\mathrm{g}, \mathrm{H} 94} \equiv C_{\mathrm{H} 94}\left(3 \Phi_{\mathrm{EUV}} / 4 \pi \alpha_{\mathrm{B}} r_{\mathrm{g}}^{3}\right)^{1 / 2}$ is that of HJLS94 derived analytically, where $\alpha_{\mathrm{B}}$ is the recombination coefficient to excited states (so-called case B) and $C_{\mathrm{H} 94} \simeq 0.2$ is the correction factor used to reproduce their numerical results. We search for the plausible base-density profile included the following process: For a given density profile, we calculate the transfer of ionizing radiation. If the gas at the disk surface $(Z=0)$ was not ionized, we reduce the base density. On the contrary, if the disk surface was well ionized, i.e., the ionizing photons have not been consumed before reaching that point, we elevate the base density. In this manner, we obtain the plausible base-density profile iteratively. In conclusion, we determine the parameters for the plausible base density to be $f=0.9$ and $p_{\text {in }}=p_{\text {out }}=1.5$, which differs from those in the HJLS94 model (Section 3). Because the outer density distribution $\left(p_{\text {out }}=1.5\right)$ is shallower than that in the HJLS94 model $\left(p_{\text {out }}=2.5\right)$, the total mass-loss rate by photoevaporation depends on total area of the evaporating annulus and thus the disk size (Section 4), unlike in the case in HJLS94.

\subsection{Radiation transfer calculation}

We adopt the gray approximation for RT, where the frequency of ionizing photons is represented by the mean value. The equation of $\mathrm{RT}$ along a ray is

$$
\frac{d I}{d s}=-(1-x) n \sigma_{\mathrm{H}} I+\frac{\alpha_{1} x^{2} n^{2}}{4 \pi} \epsilon,
$$

where $x$ is the ionization degree, $\sigma_{\mathrm{H}}$ is the cross section of a hydrogen atom, $I$ is the irradiance intensity, $\alpha_{1}$ is the radiative recombination coefficient for the ground state, and $\epsilon$ is the mean energy of ionization photons. The first term on the right-hand side represents the photon consumption by ionization, and the second term indicates the re-emission by recombinations directly to the ground state. Following HJLS94, we neglect absorption and scattering by dust grains In the case of primordial star formation, as well as present-day 
star/planet formation, this may be an appropriate assumption if grains have significantly settled toward the equatorial plane. However, the dust effects should be studied in future studies.

The ionization degree is obtained from the balance between photoionization and recombination,

$$
\frac{4 \pi x_{\mathrm{HI}} n \sigma_{\mathrm{H}} J}{\epsilon}=\alpha_{\mathrm{A}} x_{\mathrm{HII}}^{2} n^{2},
$$

where the mean intensity is

$$
J=\frac{1}{4 \pi} \int I d \Omega,
$$

and $\alpha_{\mathrm{A}}$ is the radiative recombination coefficient for all levels (so-called the case A). Solving the RT equation (equation 11) with the photoionization equilibrium (equation 12) for a given base density distribution, we search for the parameter set $\left(f, p_{\text {in }}, p_{\text {out }}\right)$ for the plausible distribution as described in Section 2.2.2.

\subsection{Numerical settings}

We conduct axisymmetric two-dimensional RT calculations in the range of stellar mass $M_{*}=10$ $-100 M_{*}$ and EUV emissivity $\Phi_{\mathrm{EUV}}=10^{49}$ $10^{51} \mathrm{~s}^{-1}$. The computational domain is a cylindrical region with radial and vertical coordinates $R<R_{\max }$ and $Z<Z_{\max }$, where $R_{\max }=Z_{\max }=$ $r_{\mathrm{g}}$ and $10 r_{\mathrm{g}}$. We assign an EUV source representing the central star at $(R, Z)=(0,0)$. Although the source radius $R_{*}$ in our calculation $(>0.2 \mathrm{AU})$ is greater than the actual stellar radius $(\lesssim 0.1 \mathrm{AU})$, we verify that the results are unchanged with varying $R_{*}$ in the range from $0.02 R_{\max }$ to $0.003 R_{\max }$. In the following section, we show the results with the highest resolution of $R_{*}=0.003 R_{\max }(0.2 \mathrm{AU}$ for $M_{*}=10 M_{\odot}$ and $\left.R_{\max }=r_{\mathrm{g}}\right)$. Because finer structures are present in the inner region, the radial and vertical grids are set to be spaced logarithmically. The EUV source radius is resolved with 10 grids and the entire computational domain by 70 grids. We solve the transfer equation by the short characteristic method along rays that are tangential to cylinders with $R=$ const. (Section 4 , Stone, Mihalas and Norman 1992). The resolution in the zenithal angle is $\Delta \theta=\pi / 1800$. Because we solve ray-tracing by tangential plane methods, the resolution of azimuthal angle changes with spatial position. We verify the accuracy of our $2 \mathrm{D}$ RT code by conducting the spherical Strömgren test with the same resolution.

\section{Results}

In this section, we present the $2 \mathrm{D}$ calculation results, which indicate that the exponent for the plausible base density is the same inside and outside the gravitational radius, $p_{\text {in }}=p_{\text {in }}=1.5$. While this value is in disagreement with the conventional value reported by HJLS94, it is consistent with the results of the radiative hydrodynamic simulation by Hosokawa et al. (2011) (Section 5.2).

First, as the fiducial case, we show the results with stellar parameters fixed at $M_{*}=10 M_{\odot}$ and $\Phi_{\mathrm{EUV}}=10^{49} \mathrm{sec}^{-1}$. From this result, we determine that the plausible base-density distribution is that with $f=0.9$ and $p_{\text {in }}=p_{\text {out }}=1.5$, for which the ionization front is located at the equatorial plane $Z=0$. Figure 2 shows the spatial profiles of ionization degree, neutral fraction, and diffuse radiation field in this case. Inside the gravitational radius ( $R<r_{\mathrm{g}} \simeq 70 \mathrm{AU}$ ), the density profile nearly agrees with that of the HJLS94 model $\left(f=1, p_{\text {in }}=1.5\right)$. However, outside the gravitational radius $\left(R>r_{\mathrm{g}}\right)$, the density determined with our method is higher; that is the disk surface $(Z=0)$ can be ionized despite a density higher than that determined by HJLS94 model.

As assumed in HJLS94, the fraction of diffuse radiation $J_{\text {diff }} / J$ is rather higher at the disk surface because of re-emission in the atmosphere (Fig. 2. bottom-left). However, this fraction reaches a maximum of $\sim 0.5$; thus, direct stellar radiation dominates in the entire region. It should be noted the diffuse radiation flux, $\boldsymbol{F}_{\text {diff }}$, has a large radial component (Figure 2, bottom-right). Such a radiation field cannot be properly expressed by $1+1 \mathrm{D}$ treatment, which emphasizes the importance of the $2 \mathrm{D}$ calculation.

Figure 3 shows the results for the most plausible density distribution and for other cases. Here, to determine the dependence on the density normalization factor $f$ and the inner density exponent $p_{\text {in }}$, the computational domain is limited inside the gravitational radius, i.e., $R_{\max }=Z_{\max }=$ $r_{\mathrm{g}}$. For a density higher than the plausible value $\left(f>0.9\right.$ or $\left.p_{\text {in }}>1.5\right)$, the ionization front does not reach the disk surface at $Z=0$, which is inconsistent with our assumption that the gas above the disk is ionized and has a high temperature. These structures cannot be in the steady state, because 

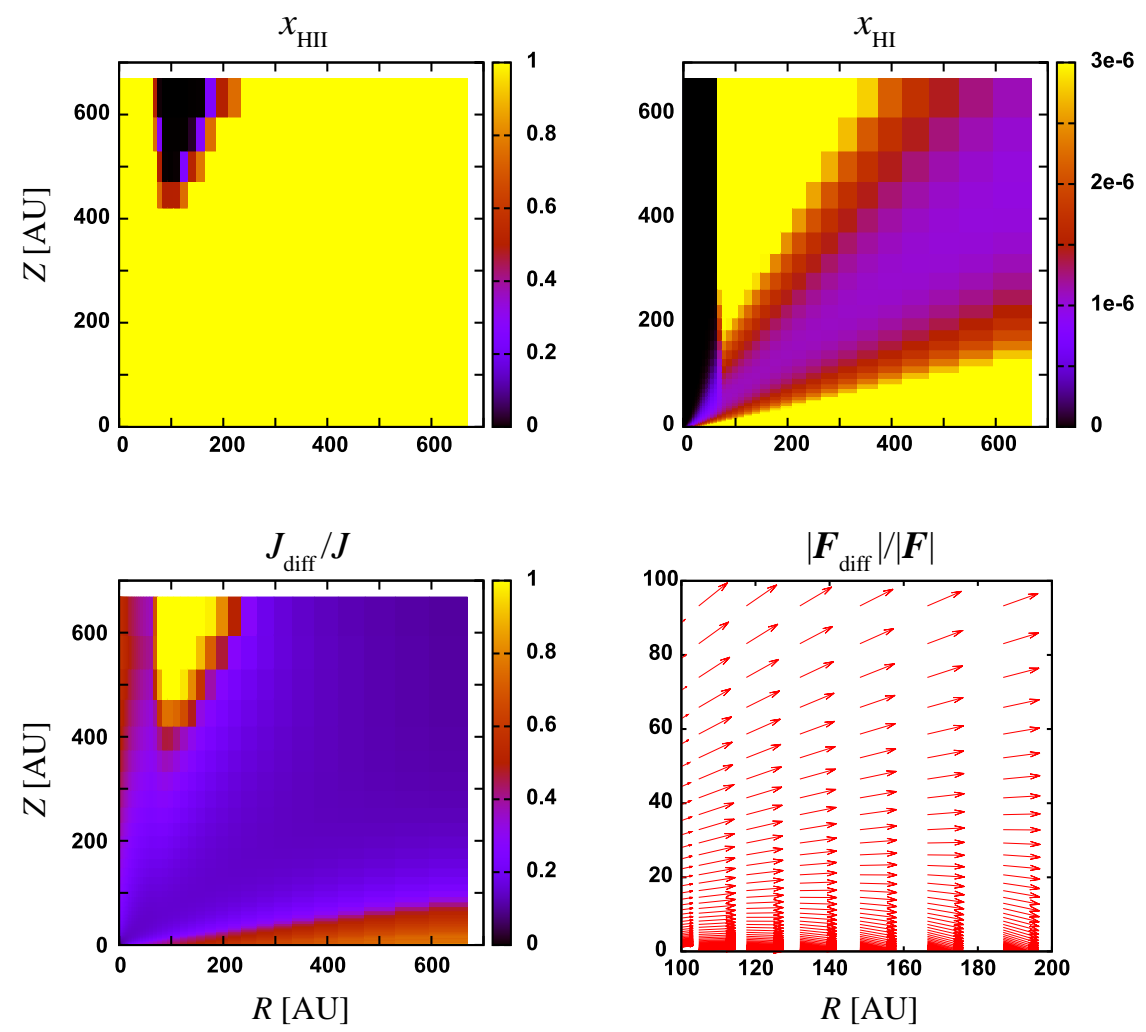

Fig. 2.- Spatial profiles of ionization degree $x_{\mathrm{HII}}$ (top-left), neutral fraction $x_{\mathrm{HI}}$ (top-right), diffuse radiation fraction $J_{\text {diff }} / J$ (bottom-left), and direction of diffuse flux $\boldsymbol{F}_{\text {diff }} /\left|\boldsymbol{F}_{\text {diff }}\right|$ (bottom-right) for the fiducial case with stellar parameters of $M_{*}=10 M_{\odot}$ and $\Phi_{\mathrm{EUV}}=10^{49} \mathrm{sec}^{-1}$, base density of $f=0.9$ and $p_{\text {in }}=p_{\text {out }}=1.5$. The gravitational radius is $r_{\mathrm{g}} \simeq 70 \mathrm{AU}$. Note that the scale on the bottom-right panel is different so as to indicate the near base profile.

the cold neutral gas would settle on the disk with the base density of ionized gas decreasing until the ionization front reaches the disk surface.

Figure 3 shows that, in all the three cases, the ionization boundary, qt which the neutral fraction abruptly increases from $x_{\mathrm{HI}} \ll 1$ to $\sim 1$, is located at the position where $\tau \sim 1$ from the central star. This fact indicates that direct stellar radiation is dominant in ionization, and the gas is ionized as far as it reaches. For the plausible distribution with $\left(f=0.9, p_{\text {in }}=p_{\text {out }}=1.5\right)$, the optical depth remains $\sim 1$ in a wide range of the radius, which indicates that the ionization front is located $Z=0$ at all radii.

To see the dependence on the outer density exponent $p_{\text {out }}$, Fig. 4 shows the neutral fraction and the optical depth for the cases with five different values of $p_{\text {out }}(0.5,1,1.5,2$, and 2.5$)$, where the other parameters are fixed at $\left(f=0.9, p_{\text {in }}=1.5\right)$. Inside the gravitational radius, both $x_{\mathrm{HI}}$ and $\tau$ behave similarly for various $p_{\text {out }}$ values, which indicates that the inner radiation field is not affected by the outer field. For $p_{\text {out }}<1.5$, the ionization front emerges above the disk when $\tau$ becomes $\simeq 1$. For $p_{\text {out }}>1.5$, the gas is completely ionized at the disk surface. Thus, the case with $p_{\text {out }}=1.5$ gives the plausible density distribution, which produces the ionization front at the disk surface.

Thus far, we have discussed cases with the stellar parameters $M_{*}=10 M_{\odot}$ and $\Phi_{\mathrm{EUV}}=$ $10^{49} \mathrm{sec}^{-1}$. The same plausible density distribution is indicated for other combinations of $\left(M_{*}\right.$, $\left.\Phi_{\text {EUV }}\right)$. Figure 5 shows the neutral fraction and optical depth for the density distribution of $f=$ $0.9, p_{\text {in }}=p_{\text {out }}=1.5$ for various stellar parameters 

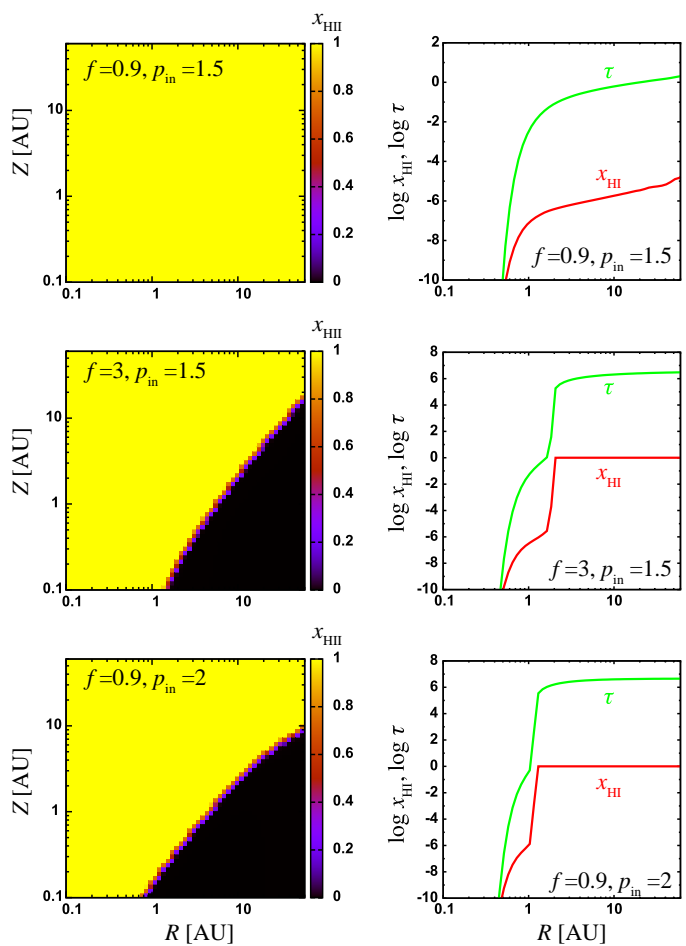

Fig. 3.- Results with application of various $f$ and $p_{\text {in }}$ showing (left) the ionization degree in the $R-Z$ plane and (right) the radial profiles of neutral fraction $\left(x_{\mathrm{HI}}\right)$ and the optical depth from the central object $(\tau)$ at the near base of $z=R_{*}$. The density parameters are $f=0.9, p_{\text {in }}=1.5$ which is the plausible case, $f=3, p_{\text {in }}=1.5$, and $f=0.9, p_{\text {in }}=2$ from top to bottom, respectively. The ionization boundary always locates at $\tau \simeq 1$.

$\left(M_{*}, \Phi_{\mathrm{EUV}}\right)$. It should be noted that the horizontal axis is the radial distance normalized by the gravitational radius. Although the neutral fraction depends on the stellar parameters, curves for the optical depth, which remains at $\simeq 1$ for a wide range in radius, completely overlap. This result indicates that the density distribution of $f=0.9$, $p_{\text {in }}=p_{\text {out }}=1.5$ is plausible for any combination of $\left(M_{*}, \Phi_{\mathrm{EUV}}\right)$.

Substituting $f=0.9$ and $p_{\text {in }}=p_{\text {out }}=1.5$ to eq.(10), we obtain the base density distribution

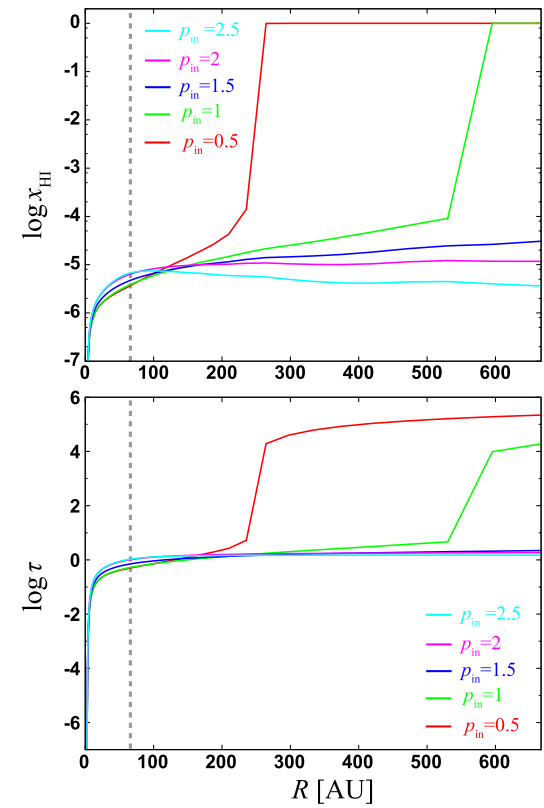

Fig. 4.- Results with application of various $p_{\text {out }}$ showing ( $t o p)$ the radial profiles of neutral fraction and (bottom) the optical depth from the central object at the near base of $Z=R_{*}$. The dashed line indicates the gravitational radius, $R=r_{\mathrm{g}}$.

model, where the density distribution follows the broken power-law with $p_{\text {in }}=1.5$ and $p_{\text {out }}=2.5$. The stellar mass does not explicitly appear in the expression of equation (14), and the base density depends on $M_{*}$ only through $\Phi_{\mathrm{EUV}}\left(M_{*}\right)$.

\section{Analytic expression for photoevapora- tion rate}

As demonstrated in the previous section, stellar radiation dominates the diffuse radiation, and the ionization front always resides near $\tau \simeq 1$. On the basis of this fact, we analytically interpret the obtained base-density profile (equation 14), and we derive a new analytical expression for the photoevaporation rate for dust-free disks.

Let us consider a ray from a star irradiating onto the disk surface (see Fig. 6). In the vicinity of the star, where

$$
\begin{aligned}
& n_{0}(R)=1.6 \times 10^{7}\left(\frac{\Phi_{\mathrm{EUV}}}{10^{49} \mathrm{~s}^{-1}}\right)^{0.5}\left(\frac{R}{10^{15} \mathrm{~cm}}\right)^{-1.5} \mathrm{~cm}^{-3} \cdot\left(14 R<R_{\mathrm{in}}=\left(r_{\mathrm{g}} R_{*}^{2}\right)^{1 / 3}\right. \\
& =0.3\left(\frac{M_{*}}{10 M_{\odot}}\right)^{1 / 3}\left(\frac{R_{*}}{0.02 \mathrm{AU}}\right)^{2 / 3}
\end{aligned}
$$




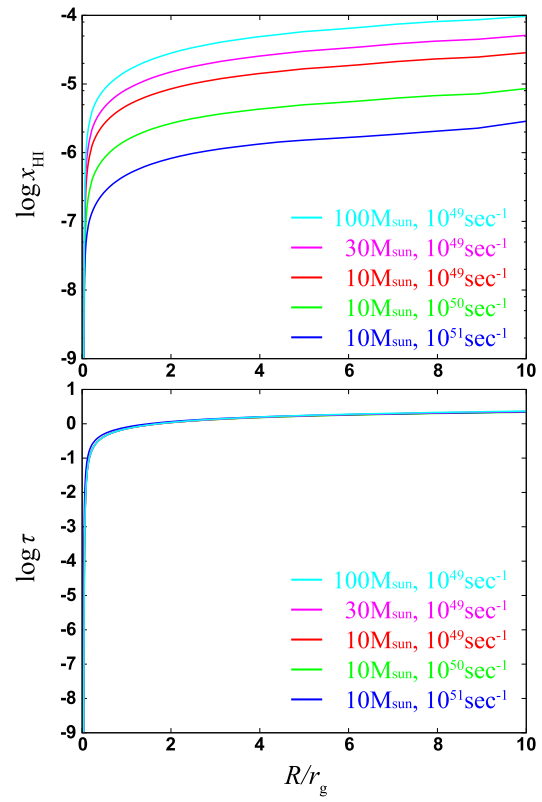

Fig. 5.- Results with application of various $M_{*}$ and $\Phi_{\text {EUV }}$ showing radial profiles of the neutral fraction and the optical depth from the central star. In the bottom panel, all curves overlap. Note that the horizontal axis is the radial distance normalized by the gravitational radius.

the scale height $H(R)$ of the ionized gas is lower than the height of the ray from the equatorial plane $\left(\sim R_{*}\right)$, and the density is low. The contribution of the gas inside $R_{\text {in }}$ to the optical depth is negligible. Outside $R_{\text {in }}$, the density along the ray is approximately given by the base density $n(R, Z) \simeq n_{0}(R)$, because the ray travels below the scale height. Then, the optical depth from the star can be written as

$$
\tau(R)=\int_{R_{\mathrm{in}}}^{R} x_{\mathrm{HI}} n_{0}\left(R^{\prime}\right) \sigma_{\mathrm{H}} d R^{\prime} .
$$

The neutral fraction $x_{\mathrm{HII}}(r, z=0)$ can be obtained from the photoionization equilibrium (equation 12),

$$
x_{\mathrm{HI}} \simeq \frac{\alpha_{\mathrm{A}} \epsilon n}{4 \pi \sigma_{\mathrm{H}} J},
$$

where the case A value $\alpha_{\mathrm{A}}$ is used as the recombination coefficient, because we consider only direct stellar radiation and ignore re-emitted diffuse radiation. In this equation, we assume that the

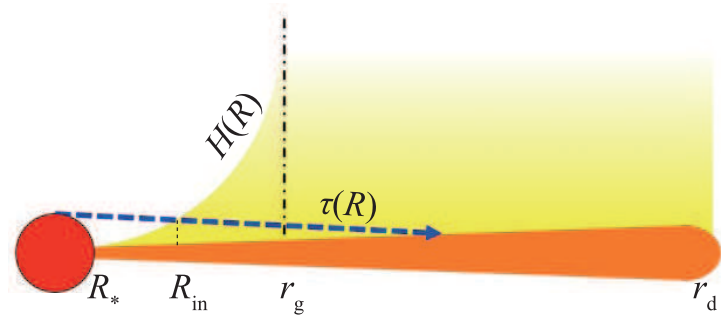

Fig. 6. - Schematic view of the light path from the central star.

neutral fraction is small $\left(x_{\mathrm{HI}} \ll 1\right)$, and the optical depth along the ray is also small $(\tau \ll 1)$; The direct stellar radiation intensity in $r \gg R_{*}$ is

$$
J_{*}=\frac{\epsilon \Phi_{\mathrm{EUV}}}{16 \pi^{2} r^{2}},
$$

which is evaluated by the radiation from the half side of the star, which considers shielding by the optically thick disk. From these values, we obtain the optical depth as,

$$
\tau(R) \simeq \int_{R_{\mathrm{in}}}^{R} \frac{4 \pi \alpha_{\mathrm{A}} n_{0}^{2} R^{\prime 2}}{\Phi_{\mathrm{EUV}}} d R^{\prime} .
$$

Assuming a single power-law distribution for the base density, $n_{0} \propto r^{-p}$, the optical depth is approximately evaluated as

$$
\tau(R) \sim \frac{4 \pi \alpha_{\mathrm{A}} n_{0}^{2} R^{3}}{\Phi_{\mathrm{EUV}}} \propto R^{3-2 p} .
$$

For the plausible density distribution, the ionization front is located at $Z=0$ for all radii or $\tau \sim 1 \propto R^{0}$. Thus, from equation (21), we obtain the exponent $p=3 / 2$. If $p<3 / 2$, the optical depth $\tau$ increases with $r$, and it does not satisfy the requisite $\tau \sim R^{0}$. Strictly speaking, with $p=3 / 2$, $\tau$ is an increasing function as $\log R$, however, it should be noted that $\log r$ is a much flatter function of $r$ than $R^{3-2 p}$ with $p<3 / 2$. In contrast, if $p>3 / 2$, the base density $n_{0}$ rapidly decreases with $R$, and the optical depth $\tau$ is dominated by the density in the inner region $\left(R \simeq R_{\text {in }}\right)$. Then, the optical depth in this case would also become constant with respect to the radius, $\tau(R) \propto R^{0}$. However, if the density is lower than the plausible value and $\tau<1$, the base density would increase because of the direct stellar radiation and ionization and would approach the plausible value, 
which has the exponent $p=3 / 2$. On the other hand, if the density is higher than the plausible value and $\tau>1$, the base density would decrease because ionizing radiation does not reach that point. Therefore, in any case for $p>3 / 2$, the density would approach the plausible value.

We can also obtain the absolute value of the base density from $\tau \simeq 1$ with $p=3 / 2$ as,

$$
\begin{aligned}
& n_{0}(R)=C\left(\frac{\Phi_{\mathrm{EUV}}}{4 \pi \alpha_{\mathrm{A}} R^{3}}\right)^{1 / 2} \\
& =1.6 \times 10^{7}\left(\frac{\Phi_{\mathrm{EUV}}}{10^{49} \mathrm{~S}^{-1}}\right)^{1 / 2}\left(\frac{R}{10^{15} \mathrm{~cm}}\right)^{-3 / 2} \mathrm{~cm}^{-3},
\end{aligned}
$$

where $C \simeq 0.4$ is the correction factor used to match our numerical result (equation 14). It is evident that the agreement between the analytical and numerical results is satisfactory.

Finally, we evaluate the photoevaporation rate by using the obtained base density from equation (22). From equation (3), the mass-loss flux from a unit area is

$$
\begin{aligned}
& \dot{\Sigma}_{\mathrm{PE}}(R)=6.0 \times 10^{-13}\left(\frac{\Phi_{\mathrm{EUV}}}{10^{49} \mathrm{sec}^{-1}}\right)^{1 / 2} \\
& \times\left(\frac{R}{1000 \mathrm{AU}}\right)^{-3 / 2} \mathrm{~g} \mathrm{~cm} \mathrm{~cm}^{-2} \mathrm{~s}^{-1} .
\end{aligned}
$$

From equation (4), the total evaporation rate from the entire disk is

$$
\begin{aligned}
& \dot{M}_{\mathrm{PE}}= 5.4 \\
& \times 10^{-5}\left(\frac{\Phi_{\mathrm{EUV}}}{10^{49} \mathrm{sec}^{-1}}\right)^{1 / 2} \\
& \times\left(\frac{r_{\mathrm{d}}}{1000 \mathrm{AU}}\right)^{1 / 2} M_{\odot \mathrm{yr}^{-1}} .
\end{aligned}
$$

Here, we assume that the disk size is significantly greater than the gravitational radius $\left(r_{\mathrm{d}} \gg r_{\mathrm{g}}\right)$. However, for very massive stars $\left(>20 M_{\odot}\right)$, the gravitational radius defined by equation (2) can be as large as the disk size. Even in this case, if we also consider effects such as radiation pressure and angular momentum in addition to gravity, the effective gravitational radius will be reduced below the disk radius (Liffman 2003; Font et al. 2004; McKee \& Tan 2008). It should be noted that our evaporation rate (equation 24) depends on the disk radius rather than the gravitational radius, unlike that given by HJLS94. This discrepancy occurs, because in the HJLS94 model, the base density steeply decreases as $R^{-5 / 2}$ outside the gravitational radius, whereas for the flat basedensity distribution $R^{-3 / 2}$ in our model, annuli near the outer radius $r_{\mathrm{d}}$ dominate the evaporation rate. Formally, equation (24) coincides with the results of HJLS94 if we change $r_{\mathrm{d}}$ to $r_{\mathrm{g}}$. Although the terms appear to differ in the expression, their numerical difference, $\sqrt{r_{\mathrm{d}} / r_{\mathrm{g}}}$, generally remains within an order of magnitude.

\section{Discussion}

Photoevaporation has an important effect on the formations of stars and planets. In this section, we discuss the impact of EUV photoevaporation on primordial star formation as well as present-day star/planetary formation by considering the new photoevaporation rate. In addition, we describe the validity and the limitations of our model.

\subsection{Impacts of photoevaporation in star/planet formation}

\subsubsection{Primordial star formation}

Recent studies have demonstrated that the protostellar accretion of primordial stars is terminated by EUV photoevaporation McKee \& Tan 2008; Hosokawa et al. 2011; Stacy et al. 2012; Hosokawa et al. 2012). Once the stellar mass exceeds $\sim 10 M_{\odot}$, the stellar surface temperature becomes sufficiently high to emit a copious amount of EUV photons. As a result, the conical $\mathrm{H}$ II regions begin to expand in the polar directions in a process known as the $\mathrm{H}$ II region breakout, which significantly decreases the infall rate from the envelope to the disk. Disk photoevaporation begins at that moment and increases with the growth of the stellar mass. Finally, accretion ceases when the growing evaporation rate reaches the infall rate such that $\dot{M}_{\mathrm{PE}}=\dot{M}_{\text {infall }}$.

In Fig 7, we show the mass of primordial stars at the termination of accretion, $\dot{M}_{\mathrm{PE}}=\dot{M}_{\text {infall }}$, estimated by our photoevaporation rate for three disk sizes of $r_{\mathrm{d}}=100,1000$, and $10000 \mathrm{AU}$ (equation (24). The disk radius is $\sim 100-10000 \mathrm{AU}$ for stars with mass of $10-1000 M_{\odot}$, as determined by the analytical model for primordial star formation developed by Tan \& McKee (2004). Here we evaluate EUV emissivity by assuming that the star is in the zero-age main sequence 


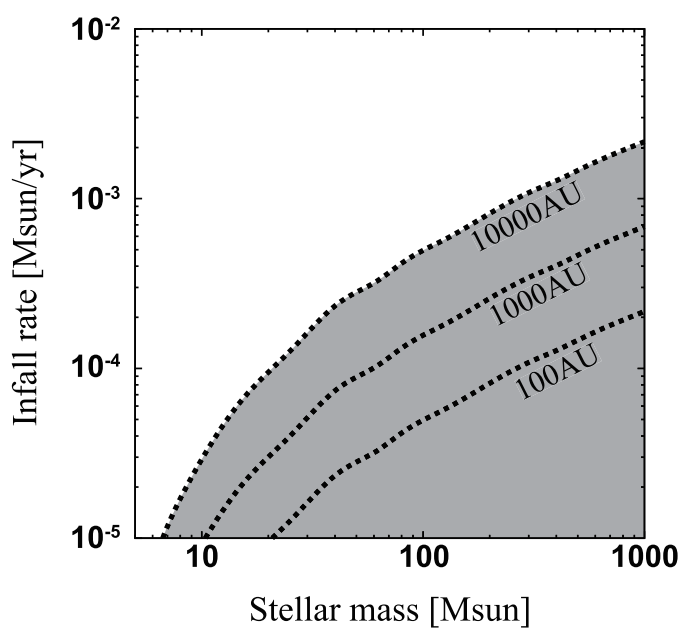

Fig. 7.- Final mass of a primordial star obtained by equating the infall rate $\dot{M}_{\text {infall }}$ with the photoevaporation rate $\dot{M}_{\mathrm{PE}}$. The three curves represent cases in which the disk radius $r_{\mathrm{d}}=100,1000$, and $10000 \mathrm{AU}$. Formation in the lower-right region is prohibited by photoevaporation. For example, in the case of $\dot{M}_{\text {infall }}=10^{-4} M_{\odot} \mathrm{yr}^{-1}$ and $r_{\mathrm{d}}=1000 \mathrm{AU}$, the final stellar mass is approximately $50 M_{\odot}$. We use the EUV emissivity $\Phi_{\mathrm{EUV}}$ of the zero-age main sequence star reported by Schaerer (2002).

(ZAMS) phase (Schaerer 2002). EUV emissivity for ZAMS stars can be approximated as $\Phi_{\mathrm{EUV}} \simeq$ $1.26 \times 10^{47}\left(M_{*} / M_{\odot}\right)^{1.4} \mathrm{sec}^{-1}$ in the range $40 M_{\odot}<$ $M_{*}<1000 M_{\odot}$. Then, we obtain the stellar mass whereby the photoevaporation rate balances the accretion rate,

$M_{*} \simeq 55\left(\frac{r_{\mathrm{d}}}{1000 \mathrm{AU}}\right)^{-0.7}\left(\frac{\dot{M}_{\text {infall }}}{10^{-4} M_{\odot} \mathrm{yr}^{-1}}\right)^{1.4} M_{\odot}$.

Here we use the typical accretion rate of $10^{-4} M_{\odot} \mathrm{yr}^{-1}$, which is smaller than the conventional rate of $\sim$ $10^{-3} M_{\odot} \mathrm{yr}^{-1}$ in primordial star formation without stellar feedback (Omukai \& Nishi 1998; Abel et al. 2002; Bromm \& Loeb 2004; Yoshida et al. 2006), because the accretion rate is reduced by approximately one order of magnitude before photoevaporation finally quenches the mass supply to the star owing to the $\mathrm{H}$ II region breakout (McKee \& Tan 2008; Hosokawa et al. 2011). The obtained mass of $55 M_{\odot}$ agrees well with the results of hydrodynamic simulations by Hosokawa et al. (2011), which indicate that the reduction of the infall rate through the $\mathrm{H}$ II region breakout, along with photoevaporation, is essential in setting the final stellar mass. It should be noted the final mass is smaller for the larger disk radius, because the evaporation rate is proportional to $r_{d}^{1 / 2}$. Therefore, stars formed in pre-stellar cores with a larger angular momentum would be smaller because of the greater radius of the protostellar disk, in addition to the possible reduction of the infall rate due to centrifugal force.

The following caveat is to be noted regarding our adoption of the ZAMS EUV emissivity: Although stars generally reach the ZAMS by the time of accretion termination, this is rather a result of reduced accretion, i.e., longer accretion time than the stellar Kelvin-Helmholtz time, through feedback during the preceding phase. In the pre-ZAMS phase, the stellar structure and the accretion rate interact in a manner such that a more rapid infall results in a larger stellar radius. Thus, EUV emissivity is smaller, which in turn, results in weaker feedback to the infall (Hosokawa et al. 2011, 2012). To treat the feedback in the pre-ZAMS phase, the elaborate modeling of stellar evolution is necessary.

\subsubsection{Present-day star/planet formation}

Before discussing present-day star/planet formation, it should be noted that our derived EUV photoevaporation rate ignores the scattering and absorption by dust grains, similar to the cases in the conventional HJLS94 model. Richling \& Yorke (1997) conducted hydrodynamical simulation to demonstrate that in some limited cases, dust scattering process increases the photoevaporation rate by a factor of $\sim 2$. We thus regard our photoevaporation rate as a rough value with a factor of a few degrees of uncertainty. Therefore, comprehensive research about the dust effect in this process is necessary.

In the formation of present-day massive stars, the maximum stellar mass may be determined by the combination of photoevaporation and radiation pressure. Precious studies have demonstrated that the longstanding issue of a radiation pressure barrier in massive star formation can be overcome by the shielding property of disk accretion (Nakano 1989; Jijina \& Adams 
1996; Krumholz et al. 2009; Kuiper et al. 2010; Tanaka \& Nakamoto 2011). However, the radiation pressure still has a strong effect in depleting the infall rate from the dusty envelope (Kuiper et al. 2012). With the depletion of the infall rate by radiation pressure, photoevaporation can terminate mass accretion onto stars. Therefore, even with an observationally claimed high accretion rate of $10^{-4}-10^{-3} M_{\odot} \mathrm{yr}^{-1}$, which is similar to that in the first star formation, the final masses of these stars are expected to be smaller than those of the first stars.

EUV photoevaporation has less importance in the dissipation of protoplanetary disks around low-mass stars. Because such stars emit few EUV photons, an increased amount of transmission radiation, such as FUV radiation and/or Xrays, is considered to dominate disk photoevaporation (Gorti \& Hollenbach 2009; Gorti et al. 2009; Owen et al. 2010, 2012). In fact, although the EUV evaporation rate determined by our model is higher than that by the HJLS94 model, the value falls below the rate determined by FUV and $\mathrm{X}$-rays by more than one order of magnitude for disks around low-mass stars. For the dissipation of protoplanetary disks, the role of UV radiation from nearby massive stars should be also be considered, because most stars are born as members of star clusters (Johnstone et al. 1998; Adams et al. 2004; Fatuzzo \& Adams 2008; Holden et al. 2011; Thompson 2013).

\subsection{Comparison with numerical simula- tions}

In this section, we discuss the validity of our model by comparing our steady density model with the numerical results reported by Hosokawa et al. (2011), who investigated photoionization feedback in primordial star formation by combining two-dimensional radiative hydrodynamics for the envelope with the stellar evolution calculation. In their simulation, direct stellar radiation is solved by ray-tracing, while diffuse radiation is treated with the flux limited diffusion approximation. The left-hand panel of Fig. 8 shows the temperature structure of the photoevaporation disk at the stellar mass $M_{*} \simeq 40 M_{\odot}$ with $\Phi_{\mathrm{EUV}} \simeq 10^{50} \mathrm{sec}^{-1}$, as determined by their fiducial model. The disk is in fact in the quasisteady state in the photoevaporation epoch. The neutral disk or troid $\left(\lesssim 10^{4} \mathrm{~K}\right)$ extends on the equatorial plane and ionized gas $\left(\gtrsim 10^{4} \mathrm{~K}\right)$ occupies the region above the disk. In their simulation, accretion is terminated by the photoevaporation at a final stellar mass of $43 M_{\odot}$.

In the right-hand panel of the figure, the density at the ionization front from the numerical simulation of Hosokawa et al. (2011) and our determined base density in the case of $p_{\text {in }}=p_{\text {out }}=1.5$ are presented as a function of the radius. In addition, the base density determined by the HJLS94 model $\left(p_{\text {in }}=1.5, p_{\text {out }}=2.5\right)$ is shown. A comparison of these values clearly reveals that our results, including the exponent of 1.5 and also the absolute value, agree with the numerical results. Thus, we conclude that our steady assumption is valid even when realistic hydrodynamical effects are included. It should be noted that our result derived with the thin-disk approximation agrees well with that of the simulation, in which the disk has finite thickness. Therefore, our model is applicable as long as direct stellar radiation irradiates the disk surface in a wide range of the radius. Our model is also consistent with results by Hosokawa et al. (2011) such that direct stellar radiation dominates photoionization.

\subsection{Limitation of our model}

It should be noted that our determined photoevaporation rate is only a rough approximation and carries some uncertainty. In our model, for example, we assume that the ionized-gas temperature is constant at $T_{\mathrm{HII}}=10^{4} \mathrm{~K}$ and that the flow velocity is given by the sound speed at $v_{\mathrm{PE}} \sim c_{\mathrm{S}, \mathrm{HII}} \propto T_{\mathrm{HII}}^{1 / 2}$. Both of these assumptions require modification in some circumstances. For the former, the ionized gas near primordial stars can exhibit higher temperatures owing to higher stellar surface temperatures and less efficient cooling ( $\sim 4 \times 10^{4} \mathrm{~K}$ in Hosokawa et al. 2011). For the latter, according to hydrodynamical simulation conducted by Font et al. (2004), the flows at the ionization boundary are slightly slower than the sound speed, which makes the mass-loss rate smaller by a factor of two. For a more accurate evaluation, sophisticated radiative hydrodynamical modeling is required.

In this study, we ignore the effects of dust grains. For solar metallicity, the scattering of 

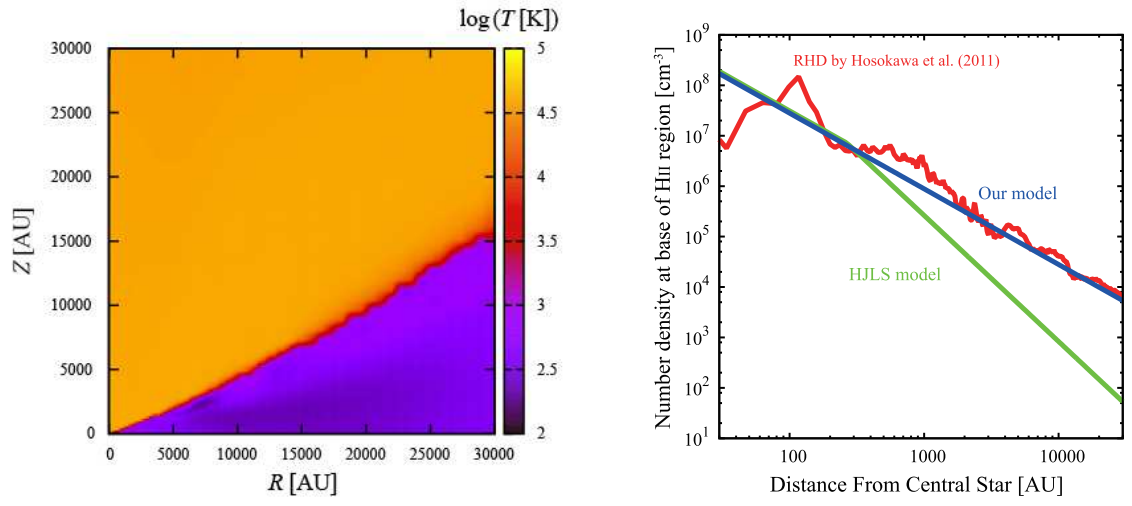

Fig. 8.- (left): Temperature distribution at the photoevaporation stage when $M_{*} \simeq 40 M_{\odot}$ and $\Phi_{\mathrm{EUV}} \simeq$ $10^{50} \mathrm{sec}^{-1}$, from the hydrodynamical simulation from Hosokawa et al. (2011). We can see the neutral disk at the equatorial plane $\left(T<10^{4} \mathrm{~K}\right)$ and the ionized region above the disk $\left(T>10^{4} \mathrm{~K}\right)$. (right): Comparison of our density models, that of HJLS94, and the numerical result by Hosokawa et al. (2011) are indicated by blue, green, and red lines, respectively. The results of our model are consistent with the numerical results.

radiation by dust in the disk atmosphere enhances the irradiation EUV flux onto the disk surface and increases the photoevaporation rate by a factor of approximately two, according to radiation hydrodynamical simulation by conducted Richling \& Yorke (1997). However, their calculation was limited to several combinations of stellar mass and disk size with the approximated RT calculation. Further calculations employing wide ranges of these parameters are needed for discussing their influence. The dependence on the disk radius is particularly interesting, because in the simulation conducted by Richling \& Yorke (1997), the mass loss from the outer region dominated the total evaporation rate.

In this study, we illustrate the case of single star formation. However, binaries or small multiples are also expected to be formed both in cases of first star formation (Machida et al. 2008; Stacy et al. 2010; Clark et al. 2011) and of present-day massive star formation (Kratter \& Matzner 2006; Krumholz et al. 2009; Peters et al. 2010). Because the infalling material is divided into multiple stars, the individual stars would be smaller than that in the single star case. This process is known as fragmentation-induced starvation (Peters et al. 2010). Therefore, the final mass estimated by equation (25) may be the upper limit; further investigation is necessary for accurate estimation.

\section{Summary}

The photoevaporation of circumstellar disks by extreme ultraviolet radiation plays an important role in star formation in the present-day as well as early universe. In this study, we revisit the limitation present in the conventional $1+1 \mathrm{D}$ approximation model developed by Hollenbach et al. (1994) in dust-free case by introducing an updated axisymmetric 2D model. Unlike that in the conventional model, the density distribution at the photoionization front located just above the disk, known as the base density' distribution, is represented by a single-exponent power law with an index of $-3 / 2$. In our model, the total photoevaporation rate depends on the outer disk radius (equation 24) in contrast to that in the conventional model, which depends on the gravitational radius. Although we have derived this base-density distribution under the steady-state assumption, we have confirmed that the results of our model are consistent with those of the radiative hydrodynamical simulation conducted by Hosokawa et al. (2011).

We are grateful to Takashi Hosokawa, Jonathan C. Tan, and Taku Takeuchi for fruitful discussions. This study is supported in part by the Grantsin-Aid by the Ministry of Education, Science and Culture of Japan (2157031:KT, 21540434, 24340102:TN, 2124402, 21684007:KO). This study 
is also supported by Global COE program "From the Earth to Earths.h

\section{REFERENCES}

Abel, T., Bryan, G. L., \& Norman, M. L. 2002, Science, 295, 93

Adams, F. C., Hollenbach, D., Laughlin, G., \& Gorti, U. 2004, ApJ, 611, 360

Armitage, P. J. 2011, ARA\&A, 49, 195

Bally, J., \& Scoville, N. Z. 1982, ApJ, 255, 497

Bromm, V., Coppi, P. S., \& Larson, R. B. 1999, ApJ, 527, L5

Bromm, V., \& Loeb, A. 2004, New A, 9, 353

Clarke, C. J., Gendrin, A., \& Sotomayor, M. 2001, MNRAS, 328, 485

Clark, P. C., Glover, S. C. O., Smith, R. J., et al. 2011, Science, 331, 1040

Fatuzzo, M., \& Adams, F. C. 2008, ApJ, 675, 1361

Font, A. S., McCarthy, I. G., Johnstone, D., \& Ballantyne, D. R. 2004, ApJ, 607, 890

Gorti, U., \& Hollenbach, D. 2009, ApJ, 690, 1539

Gorti, U., Dullemond, C. P., \& Hollenbach, D. 2009, ApJ, 705, 1237

Holden, L., Landis, E., Spitzig, J., \& Adams, F. C. 2011, PASP, 123, 14

Hollenbach, D., Johnstone, D., Lizano, S., \& Shu, F. 1994, ApJ, 428, 654 (HJLS94)

Hosokawa, T., Omukai, K., Yoshida, N., \& Yorke, H. W. 2011, Science, 334, 1250

Hosokawa, T., Yoshida, N., Omukai, K., \& Yorke, H. W. 2012, ApJ, 760, L37

Jijina, J., \& Adams, F. C. 1996, ApJ, 462, 874

Johnstone, D., Hollenbach, D., \& Bally, J. 1998, ApJ, 499, 758

Kratter, K. M., \& Matzner, C. D. 2006, MNRAS, 373,1563

Kraus, S., Hofmann, K.-H., Menten, K. M., et al. 2010, Nature, 466, 339
Krumholz, M. R., Klein, R. I., McKee, C. F., Offner, S. S. R., \& Cunningham, A. J. 2009, Science, 323, 754

Kuiper, R., Klahr, H., Beuther, H., \& Henning, T. 2010, ApJ, 722, 1556

Kuiper, R., Klahr, H., Beuther, H., \& Henning, T. 2012, A\&A, 537, A122

Liffman, K. 2003, PASA, 20, 337

Machida, M. N., Omukai, K., Matsumoto, T., \& Inutsuka, S.-i. 2008, ApJ, 677, 813

McKee, C. F., \& Tan, J. C. 2008, ApJ, 681, 771

Nakano, T. 1989, ApJ, 345, 464

Omukai, K., \& Nishi, R. 1998, ApJ, 508, 141

Omukai, K., \& Palla, F. 2003, ApJ, 589, 677

Owen, J. E., Ercolano, B., Clarke, C. J., \& Alexander, R. D. 2010, MNRAS, 401, 1415

Owen, J. E., Clarke, C. J., \& Ercolano, B. 2012, MNRAS, 422, 1880

Peters, T., Klessen, R. S., Mac Low, M.-M., \& Banerjee, R. 2010, ApJ, 725, 134

Richling, S., \& Yorke, H. W. 1997, A\&A, 327, 317

Schaerer, D. 2002, A\&A, 382, 28

Shu, F. H., Johnstone, D., \& Hollenbach, D. 1993, Icarus, 106, 92

Stacy, A., Greif, T. H., \& Bromm, V. 2010, MNRAS, 403, 45

Stacy, A., Greif, T. H., \& Bromm, V. 2012, MNRAS, 422, 290

Stone, J. M., Mihalas, D., \& Norman, M. L. 1992, ApJS, 80, 819

Tan, J. C., \& McKee, C. F. 2004, ApJ, 603, 383

Tanaka, K. E. I., \& Nakamoto, T. 2011, ApJ, 739, L50

Thompson, T. A. 2013, MNRAS, 431, 63

Yoshida, N., Omukai, K., Hernquist, L., \& Abel, T. 2006, ApJ, 652, 6

This 2-column preprint was prepared with the AAS LATEX macros v5.2. 